\title{
Terahertz oscillation of resonant tunneling diodes with deep and thin quantum wells
}

\author{
Hidetoshi Kanaya, Safumi Suzuki, and Masahiro Asada ${ }^{\text {a) }}$ \\ Interdisciplinary Graduate School of Science and Engineering, Tokyo Institute of \\ Technology, 2-12-1-S9-3 Ookayama, Meguro-ku, Tokyo 152-8552, Japan \\ a) asada@pe.titech.ac.jp
}

Abstract: Terahertz oscillators using AlAs/InGaAs resonant tunneling diodes with deep and thin quantum wells are reported. Although a thin well has been shown to be effective for highfrequency oscillation until now due to a reduced electron dwell time, it caused an increase in bias voltage. We introduce a deep well with indium-rich InGaAs to maintain or even to reduce the bias voltage. Current-voltage and oscillation characteristics are compared between the quantum wells with 3.5-nm-thick $\mathrm{In}_{0.8} \mathrm{Ga}_{0.2} \mathrm{As}$ and 3 -nm-thick $\mathrm{In}_{0.9} \mathrm{Ga}_{0.1}$ As. The highest oscillation frequency was $0.96 \mathrm{THz}$ for the former while $1.27 \mathrm{THz}$ for the latter without increase in bias voltage. Keywords: terahertz oscillators, resonant tunneling diode, slot antenna, terahertz integrated circuits, electron dwell time, quantum well

Classification: Electron devices, circuits, and systems

\section{References}

[1] M. Tonouchi: Nat. Photonics 1 (2007) 97.

[2] T. Nagatsuma: Electron. Express 8 (2011) 1127.

[3] H. Song and T. Nagatsuma: IEEE Trans. Terahertz Sci. Technol. 1 (2011) 256.

[4] H. J. Song, A. Ajito, Y. Muramoto, A. Wakatsuki, T. Nagatsuma and N. Kukutsu: Electron. Lett. 48 (2012) 953.

[5] K. Ishigaki, M. Shiraishi, S. Suzuki, M. Asada, N. Nishiyama and S. Arai: Electron. Lett. 48 (2012) 582.

[6] R. Köhler, A. Tredicucci, F. Beltram, H. E. Beere, E. H. Linfeld, A. G. Davies, D. A. Ritchie, R. C. Iotti and F. Rossi: Nature 417 (2002) 156.

[7] B. S. Williams: Nat. Photonics 1 (2007) 517.

[8] S. Fathololoumi, E. Dupont, C. W. I. Chan, Z. R. Wasilewski, S. R. Laframboise, D. Ban, A. Matyas, C. Jirauschek, Q. Hu and H. C. Liu: Opt. Exp. 20 (2012) 3866.

[9] V. Radisic, L. Samoska, W. R. Deal, X. B. Mei, W. Yoshida, P. H. Liu, J. Uyeda, A. Fung, T. Gaier and R. Lai: MTT-S Int. Microwave Symp. (2008) 395.

[10] M. Seo, M. Urteaga, J. Hacker, A. Young, Z. Griffith, V. Jain, R. Pierson, P. Rowell, A. Skalare, A. Peralta, R. Lin, D. Pukala and M. Rodwell: IEEE J. Solid-State Circuits 46 (2011) 2203. 
[11] O. Momeni and E. Afshari: IEEE J. Solid-State Circuits 46 (2011) 583.

[12] Y. M. Tousi, O. Momeni and E. Afshari: IEEE J. Solid-State Circuits 47 (2012) 3032.

[13] E. R. Brown, J. R. Sönderström, C. D. Parker, L. J. Mahoney, K. M. Molvar and T. C. McGill: Appl. Phys. Lett. 58 (1991) 2291.

[14] M. Reddy, S. C. Martin, A. C. Molnar, R. E. Muller, R. P. Smith, P. H. Siegel, M. J. Mondry, M. J. W. Rodwell, H. Kroemer and S. J. Allen: IEEE Electron Device Lett. 18 (1997) 218.

[15] M. Asada, S. Suzuki and N. Kishimoto: Jpn. J. Appl. Phys. 47 (2008) 4375.

[16] S. Suzuki, M. Asada, A. Teranishi, H. Sugiyama and H. Yokoyama: Appl. Phys. Lett. 97 (2010) 242102.

[17] M. Feiginov, C. Sydlo, O. Cojocari and P. Meissner: Appl. Phys. Lett. 99 (2011) 233506.

[18] H. Kanaya, H. Shibayama, R. Sogabe, S. Suzuki and M. Asada: Appl. Phys. Express 5 (2012) 124101.

[19] Y. Koyama, R. Sekiguchi and T. Ouchi: Appl. Phys. Express 6 (2013) 064102 .

[20] M. Shiraishi, H. Shibayama, K. Ishigaki, S. Suzuki, M. Asada, H. Sugiyama and H. Yokoyama: Appl. Phys. Express 4 (2011) 064101.

[21] S. Suzuki, M. Shiraishi, H. Shibayama and M. Asada: IEEE J. Sel. Topics Quantum Electron. 19 (2013) 8500108.

[22] Main part of this work was presented at; H. Kanaya, S. Suzuki and M. Asada: Int. Symp. Frontier of Terahertz Technol. (2012) Pos. 1. 14.

[23] A. Teranishi, S. Suzuki, K. Shizuno, M. Asada, H. Sugiyama and H. Yokoyama: IEICE Trans. Electron. E95-C (2012) 401.

\section{Introduction}

Recently, the terahertz $(\mathrm{THz})$ frequency range has received considerable attention because it can be employed in various applications [1]. In particular, high-capacity short-distance wireless communication is an important application of this range. Demonstrations of $\mathrm{THz}$ communication have been intensively carried out $[2,3,4,5]$.

Compact and coherent solid-state sources are considered to be key components for such an application. Because the $\mathrm{THz}$ range exists between light waves and millimeter waves, both optical and electronic devices are being investigated as $\mathrm{THz}$ sources. $\mathrm{THz}$ quantum cascade lasers for semiconductor single oscillators have been studied from the optical device side $[6,7,8]$. On the electron device side, oscillators with heterostructure bipolar transistors (HBTs), high electron mobility transistors (HEMTs), and Si CMOS transistors are being studied intensively as $\mathrm{THz}$ sources $[9$, 10, 11, 12]. Resonant tunneling diodes (RTDs) are also a good candidate $[13,14,15]$. High-frequency and high-power oscillation has been achieved at room temperature [16, 17, 18, 19, 20, 21].

For high-frequency oscillation in RTDs, it is important to reduce the electron dwell time in the resonant tunneling structure [15]. We reported fundamental oscillation up to $1.3 \mathrm{THz}$ in RTDs with thin quantum wells in which the electron dwell time was reduced [18]. Although thin wells are 
effective for high frequency oscillation, the bias voltage for the negative differential conductance increases due to the elevation of the resonance levels in the well. The bias voltage must be reduced to improve device efficiency and also to avoid a high electric field in the collector spacer layer, which causes various phenomena degrading the oscillation characteristics, such as the increase in electron scattering, temperature increase due to hotelectron generation, and the break down. In this letter, we introduce indium-rich InGaAs to the well layer in order to keep or even to reduce the bias voltage due to depression of the well bottom. The effects of thin and deep wells on current-voltage and oscillation characteristics are discussed [22].

\section{Device structure}

Figure 1 shows the schematic structure of the RTD oscillator integrated with a slot antenna. An AlAs/InGaAs double-barrier RTD was located at the center of a slot antenna on a semi-insulating InP substrate. A standing wave of the electromagnetic field is formed along the slot antenna; thus, the antenna acts as a resonator while also radiates output power. The electrodes of the RTD are connected to the upper and lower electrodes of the antenna. At both edges of the slot, the electrodes are overlapped with a $\mathrm{SiO}_{2}$ layer between them. This structure is used to form reflectors of highfrequency electromagnetic waves and to simultaneously achieve DC bias separation. A parallel resistance element made of a bismuth film is connected outside the antenna electrodes to suppress parasitic oscillations at $2-3 \mathrm{GHz}$ caused by the resonance formed by the external circuits, including the bias-supplying lines.

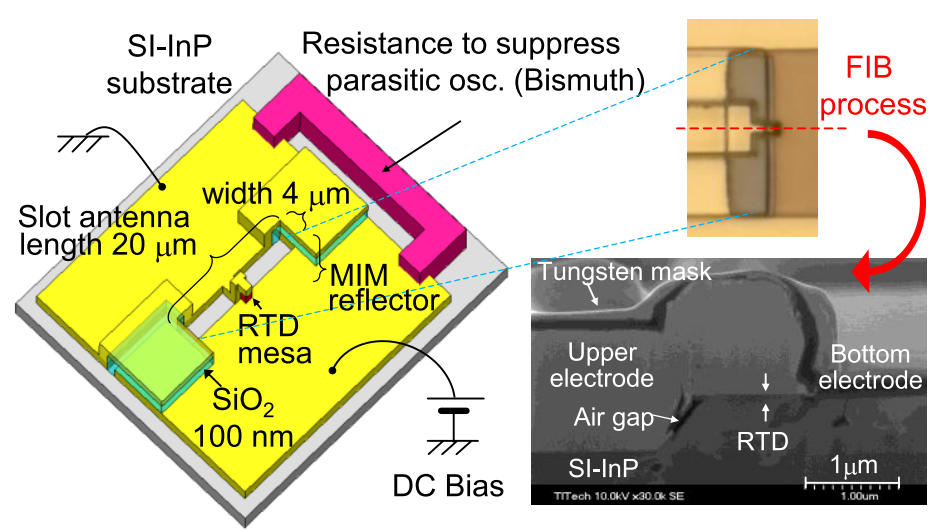

Fig. 1. Schematic structure of RTD oscillator with micrograph and cross-sectional scanning-electron-microscope image of fabricated device.

Oscillations take place if the negative differential conductance (NDC) of the RTD, $G_{R T D}$, compensates for the radiation loss of the antenna. $G_{R T D}$ decreases with the increase in frequency as a result of the intrinsic delay time $\tau$ of electrons in RTD, which consists of the dwell time in the resonant tunneling layers (double barriers and quantum well) and the transit time across the collector depletion region [15]. $G_{R T D}$ is expressed as $G_{R T D}=(3 / 2)$ $\Delta I / \Delta V \cos \omega \tau$, where $\Delta I$ and $\Delta V$ are the current and voltage widths of the NDC region (see Fig. 3), and $\omega$ is the angular frequency. The output power 
is proportional to $\cos \omega \tau \times \Delta I \Delta V$. High frequency and high power operation is achieved with a small $\tau$ and large $\Delta I$ and $\Delta V$ with keeping $\Delta I / \Delta V$. Details on the analysis of the oscillation frequency and output power are described in [15].

Figure 2 shows the layer structure of the fabricated RTD. The schematic band diagram is also shown on the right-hand side of Fig. 2. These layers were grown on a semi-insulating InP substrate using molecular beam epitaxy. The electrons flow from the top to the bottom. A cap layer with an indium-rich strained composition and high doping concentration was used to reduce the contact resistance of the electrode.

\begin{tabular}{|c|c|c|c|}
\hline $\mathrm{n}+-\mathrm{In}_{0.7} \mathrm{Ga}_{0.3} \mathrm{As}$ & \multicolumn{2}{|c|}{$8 \mathrm{~nm} \quad$ (Doping $\left.2 \times 10^{19} \mathrm{~cm}^{-3}\right)$} & \\
\hline $\mathrm{n}+-\mathrm{In}_{0.53} \mathrm{Ga}_{0.47} \mathrm{As}$ & \multicolumn{2}{|c|}{$15 \mathrm{~nm}$ (Doping $\left.2 \times 10^{19} \mathrm{~cm}^{-3}\right)$} & \\
\hline un- $\ln _{0.53} \mathrm{Ga}_{0.47} \mathrm{As}$ & \multicolumn{2}{|l|}{ 25nm Spacer } & \\
\hline AlAs & 1.0nm Barrier & & \\
\hline $\begin{array}{c}\text { un- } \ln _{0.8} G_{0.2} \text { As } 3.5 \mathrm{~nm} \\
\text { or un- } \ln _{0.9} G_{0.1} \text { As } 3.0 \mathrm{~nm}\end{array}$ & Well & RTD & \\
\hline AlAs & 1.0nm Barrier & & \\
\hline un- $\ln _{0.47} \mathrm{Ga}_{0.53} \mathrm{As}$ & \multicolumn{2}{|l|}{$2 \mathrm{~nm}$ Spacer } & \\
\hline $\mathrm{n}-\mathrm{In}_{0.49} \mathrm{Ga}_{0.51} \mathrm{As}$ & \multicolumn{2}{|c|}{$2.5 \mathrm{~nm}$ (Doping $\left.3 \times 10^{18} \mathrm{~cm}^{-3}\right)$} & \\
\hline $\mathrm{n}-\mathrm{In}_{0.51} \mathrm{Ga}_{0.49} \mathrm{As}$ & \multicolumn{2}{|c|}{$2.5 \mathrm{~nm}$ (Doping $\left.3 \times 10^{18} \mathrm{~cm}^{-3}\right)$} & e \\
\hline $\mathrm{n}-\ln _{0.53} \mathrm{Ga}_{0.47} \mathrm{As}$ & \multicolumn{2}{|c|}{ 20nm (Doping $3 \times 10^{18} \mathrm{~cm}^{-3}$ ) } & \\
\hline $\mathrm{n}+-\operatorname{In}_{0.53} \mathrm{Ga}_{0.47} \mathrm{As}$ & \multicolumn{2}{|c|}{$400 \mathrm{~nm}$ (Doping $\left.2 \times 10^{19} \mathrm{~cm}^{-3}\right)$} & \\
\hline un- $\ln _{0.53} \mathrm{Ga}_{0.47} \mathrm{As}$ & \multicolumn{2}{|c|}{$200 \mathrm{~nm}$} & \\
\hline SI-InP Sub. & & \\
\hline
\end{tabular}

Fig. 2. Layer structure of the RTDs with two different well strcutures. A band diagram is also shown.

A reduction in the electron dwell time was expected in a thin quantum well through an increase in the width of the tunneling transmission coefficient, because the dwell time is inversely proportional to this width. However, a high bias voltage was required due to the increase in resonance level. Depression of the bottom of the quantum well with indium-rich InGaAs is effective in decreasing the bias voltage. In this work, we prepared two RTDs with different quantum well layers; 3.5-nm-thick $\operatorname{In}_{0.8} \mathrm{Ga}_{0.2} \mathrm{As}$ and 3-nm-thick $\operatorname{In}_{0.9} \mathrm{Ga}_{0.1}$ As (thin and deep well), as shown in Fig. 2. The former composition is the same as that in the previous reports $[16,18,20$, $21,22]$, which the latter is newly introduced here.

\section{Oscillation characteristics}

Figure 3 shows the $I-V$ characteristics for the two RTDs mentioned above. The voltage at the current peak without the deep and thin well was $0.31 \mathrm{~V}$, whereas a similar but slightly reduced peak voltage of $0.28 \mathrm{~V}$ was obtained for the deep and thin well despite the decreased well thickness. We also obtained $\Delta J$ (the density of $\Delta I$ ) and $\Delta V$ as $5.4 \mathrm{~mA} / \mu \mathrm{m}^{2}$ and $0.32 \mathrm{~V}$ for the RTD without the deep and thin well, while $5.3 \mathrm{~mA} / \mu \mathrm{m}^{2}$ and $0.43 \mathrm{~V}$ for the deep and thin well.

We fabricated RTD oscillators integrated with a $20-\mu \mathrm{m}$-long slot antenna. Fig. 4 shows the dependence of the fundamental oscillation frequency on mesa area for RTDs with and without deep and thin well. For both RTDs, the oscillation frequency increased with decreasing mesa area due to the decrease in capacitance. We obtained the fundamental 


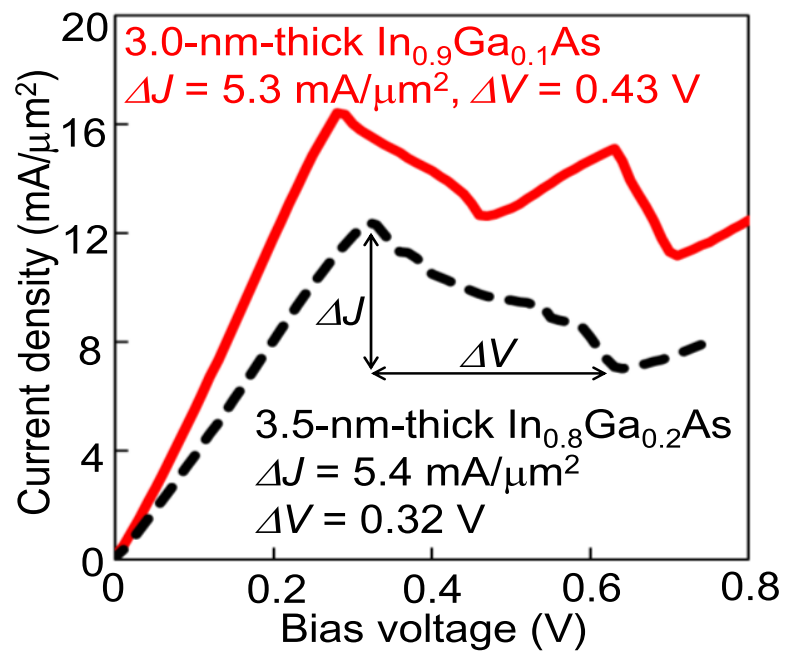

Fig. 3. $I-V$ characteristics for the RTDs with and without deep quantum well.

oscillation up to 1.27 and $0.96 \mathrm{THz}$ with and without the deep and thin well, respectively. The highest oscillation frequency in this experiment was $1.27 \mathrm{THz}$ at the mesa area of $0.25 \mu \mathrm{m}^{2}$. The output power of the device oscillating at $1.27 \mathrm{THz}$ was $\sim 1 \mu \mathrm{W}$.

Because the absolute value of $G_{R T D}$ decreases with the increase in oscillation frequency due to the intrinsic delay and the decrease in mesa area, there was a limit to the mesa area below which the oscillation could not be obtained due to insufficient $G_{R T D}$ for the compensation of the radiation loss. This limit of the mesa area is smaller for a thinner well, because $G_{R T D}$ less degraded due to the shorter dwell time. Due to this condition, the higher frequency oscillation was obtained in the deep and thin well in Fig. 4, as mentioned above. The oscillation frequency at the same area was also higher for the deep and thin well, because the additional capacitance generated by the delay time including the dwell time [15] is smaller.

We estimated the intrinsic delay time by fitting the theoretical calculation to the experimental results in Fig. 4 with the same analysis as that in [23]. Although we took into account the parasitic elements in this

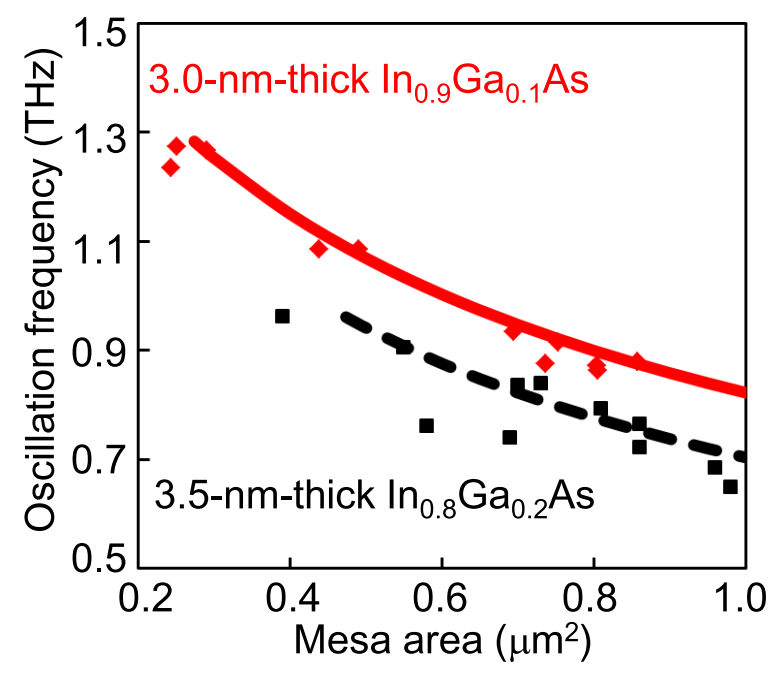


analysis, the dependence of oscillation frequency on intrinsic delay time is roughly expressed as follows. The oscillation frequency is mainly determined by the parallel resonance of the $L C$ circuit constructed with the RTD and antenna as $1 / 2 \pi \sqrt{L\left(C_{a n t}+C_{R T D}+C_{d e p}\right)}$, where $L$ is the inductance of the antenna, $C_{a n t}, C_{R T D}$, and $C_{d e p}$ are the antenna capacitance, additional capacitance due to the delay time, and depletion layer capacitance of the RTD, respectively. $C_{R T D}$ is expressed as $\tau G_{R T D \text {. }}$, through which the oscillation frequency depends on intrinsic delay time. $L$ and $C_{\text {ant }}$ of $20 \mu \mathrm{m}$ antenna were calculated as $4 \mathrm{pH}$ and $1 \mathrm{fF}$, respectively, with the three-dimensional electromagnetic simulator (ANSYS HFSS). $C_{d e p}$ was estimated as 8 and $11 \mathrm{fF} / \mu \mathrm{m}^{2}$, and $G_{R T D}$ was 19 and $25 \mathrm{fF} / \mu \mathrm{m}^{2}$ for the RTDs with and without the deep and thin well, respectively. The fitting curves are also shown in Fig. 4. The estimated intrinsic delay time $\tau$ was 40 and $110 \mathrm{fs}$ for the RTDs with and without the deep and thin well, respectively.

Figure 5 shows the output power as a function of the oscillation frequency for the RTDs with and without the deep and thin well. The theoretical curves calculated with the analysis in [15] were also plotted. In the theoretical curves, the expected output power is larger for the deep and thin well at the same frequency, because of the larger $\Delta V$ and mesa area. In the experimental result, however, the output power below about $1 \mathrm{THz}$ was almost the same between the two RTDs. The reason for the discrepancy between the theory and experiment is not clear at present. Although deviation of the parameter values used in the calculation, such as the contact resistance, may be a possible cause, a detailed discussion is necessary. Higher-frequency oscillation with higher output power will be further expected for structure optimization in terms of the thickness and material in the well and spacer layer.

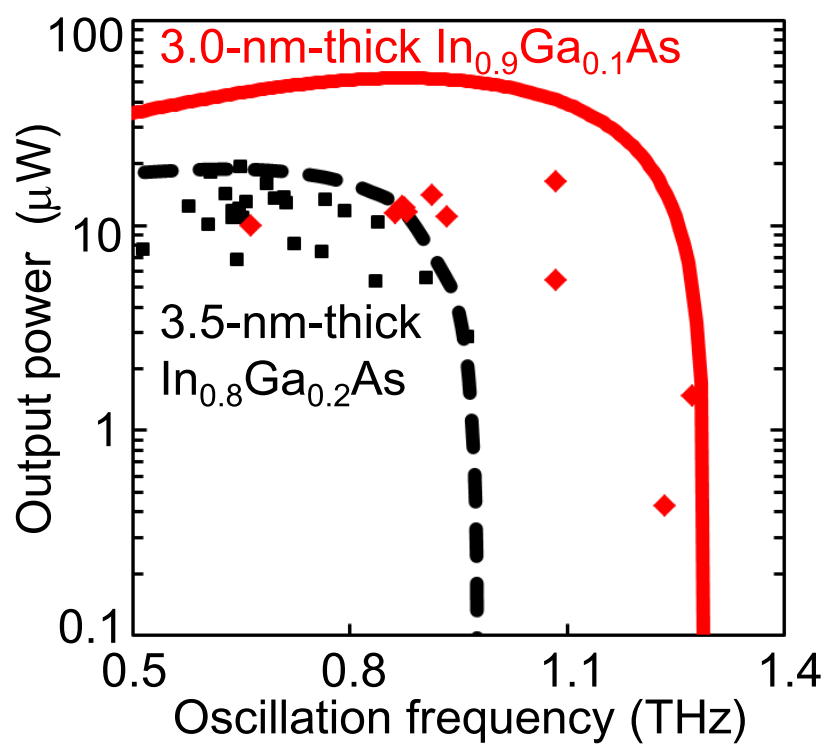

Fig. 5. Experimental and theoretical output power as a function of oscillation frequency for the RTDs with and without deep and thin quantum well. 


\section{Conclusions}

We reported on THz oscillators using AlAs/InGaAs RTDs with deep and thin quantum wells. Although a thin well has been shown to be effective for high-frequency oscillation until now due to a reduced electron dwell time, it caused an increase in bias voltage because of the elevation of the resonance levels. In this work, we introduced a deep well with indium-rich InGaAs to maintain or even to reduce the bias voltage. The $I-V$ and oscillation characteristics were compared between the quantum wells with 3.5-nmthick $\mathrm{In}_{0.8} \mathrm{Ga}_{0.2}$ As and 3-nm-thick $\mathrm{In}_{0.9} \mathrm{Ga}_{0.1}$ As. The latter is the thin and deep well. In the $I$ - $V$ characteristics, the voltage at the current peak was slightly lower for the thin and deep well. The highest oscillation frequency obtained in this experiment was $1.27 \mathrm{THz}$ and $0.96 \mathrm{THz}$ for the RTDs with and without thin and deep well, respectively. These results indicate that the thin and deep well is effective for high-frequency oscillation of RTDs without increase in bias voltage, and will be useful for compact and highefficiency $\mathrm{THz}$ oscillators with RTDs.

\section{Acknowledgments}

We thank Emeritus Professors Y. Suematsu and K. Furuya of the Tokyo Institute of Technology for their continuous encouragement. We also thank Professors S. Arai and Y. Miyamoto and Associate Professors M. Watanabe, and N. Nishiyama of the Tokyo Institute of Technology for fruitful discussions and encouragement. This work was supported by the Scientific Grants-in-Aid from the Ministry of Education, Culture, Sports, Science and Technology, Japan; the Industry-Academia Collaborative R\&D Program from the Japan Science and Technology Agency, Japan; and the Strategic Information and Communications R\&D Promotion Programme from the Ministry of Internal Affairs and Communications. 